\title{
P-III hemorrhagic metalloproteinases from Russell's viper venom: Cloning, characterization, phylogenetic and functional site analyses ${ }^{\text {it }}$
}

\author{
Hong-Sen Chen ${ }^{a}$, Hsin-Yu Tsai ${ }^{b}$, Ying-Ming Wang ${ }^{\text {b }}$, Inn-Ho Tsai ${ }^{\text {a,b,* }}$ \\ ${ }^{a}$ Graduate Institute of Biochemical Sciences, National Taiwan University, Taipei, Taiwan \\ ${ }^{\mathrm{b}}$ Institute of Biological Chemistry, Academia Sinica, Taipei, Taiwan
}

Received 25 December 2007; accepted 14 May 2008

Available online 23 May 2008

\begin{abstract}
Two homologous P-III hemorrhagic metalloproteinases were purified from Russell's viper venoms from Myanmar and Kolkata (eastern India), and designated as daborhagin-M and daborhagin-K, respectively. They induced severe dermal hemorrhage in mice at a minimum hemorrhagic dose of $0.8-0.9 \mu \mathrm{g}$. Daborhagin-M specifically hydrolyzed an A $\alpha$-chain of fibrinogen, fibronectin, and type IV collagen in vitro. Analyses of its cleavage sites on insulin chain $\mathrm{B}$ and kinetic specificities toward oligopeptides suggested that daborhagin-M prefers hydrophobic residues at the $\mathrm{P}_{1}, \mathrm{P}_{1}{ }^{\prime}$, and $\mathrm{P}_{2}{ }^{\prime}$ positions on the substrates. Of the eight Daboia geographic venom samples analyzed by Western blotting, only those from Myanmar and eastern India showed a strong positive band at $65 \mathrm{kDa}$, which correlated with the high risk of systemic hemorrhagic symptoms elicited by Daboia envenoming in both regions. The full sequence of daborhagin-K was determined by cDNA cloning and sequencing, and then confirmed by peptide mass fingerprinting. Furthermore, molecular phylogenetic analyses based on 27 P-IIIs revealed the co-evolution of two major P-III classes with distinct hemorrhagic potencies, and daborhagin-K belongs to the most hemorrhagic subclass. By comparing the absolute complexity profiles between these two classes, we identified four structural motifs probably responsible for the phylogenetic subtyping and hemorrhagic potencies of P-III SVMPs.
\end{abstract}

(C) 2008 Elsevier Masson SAS. All rights reserved.

Keywords: metalloproteinase; Substrate specificity; Geographic variation; Phylogenetic analysis; Daboia russelii; Daboia siamensis

\section{Introduction}

Local and systemic hemorrhages are prominent symptoms of envenoming by snakes of the Viperidae family [1]. Symptoms observed are caused mainly by the actions of snake venom metalloproteinases (SVMPs), which belong to the reprolysin subfamily of metzincins [2]. SVMP-induced hemorrhage may result from fibrinogenolysis that impairs coagulation, and the degradation of basement membrane proteins,

\footnotetext{
Abbreviations: MHD, minimum hemorrhagic dose; MMP, matrix metalloproteinase; PMF, peptide mass fingerprinting; SVMPs, snake venom metalloproteinases.

th The nucleotide sequence of daborhagin-K was deposited in GenBank with the accession number DQ137798.

* Corresponding author. Institute of Biological Chemistry, Academia Sinica, P.O. Box 23-106, Taipei, Taiwan. Tel.: +88622362 0264; fax: +8862 23635038.

E-mail address: bc201@gate.sinica.edu.tw (I.-H. Tsai).
}

which damages blood vessels $[3,4]$. SVMPs are categorized into P-I, P-II, and P-III groups according to the extension of several structural domains [2]. P-I enzymes comprise only the metalloproteinase domain, P-II enzymes contain a disintegrin domain after the metalloproteinase domain, and P-III enzymes $(50-70 \mathrm{kDa})$ are usually glycoproteins that contain an additional Cys-rich C-terminal domain. In general, the PIII SVMPs are more hemorrhagic than the P-I SVMPs, which indicate that the additional domains of P-IIIs might contribute to their hemorrhagic potencies.

In addition to hemorrhage, other versatile functions have also been reported for P-III SVMPs, e.g. activating prothrombin [5,6], inducing endothelial cell apoptosis [7], cleaving integrins [3], and inhibiting platelet functions [8], which implies that more structural diversities exist between P-III SVMPs. Based on the position of the seventh cysteinyl residue in the metalloproteinase domain, three new P-III subclasses were 
revealed [2,9]: P-IIIa, which undergoes autoproteolysis to release a $\sim 30-\mathrm{kDa}$ fragment with disintegrin- and Cys-rich domains; P-IIIb, which forms a dimeric structure; and P-IIIc, which contains the seventh cysteinyl residue at position 100 in its metalloproteinase domain. However, the structural elements attributed to their functional variations and hemorrhagic potencies remain elusive [2].

Two species of Russell's vipers, Daboia siamensis and Daboia russelii, are medically the most important Viperinae in South and Southeast Asia [10], and distributed in eastern and western ranges of their habitat, respectively [11]. Consumptive coagulopathy resulting in spontaneous and systemic bleeding are the main causes of fatal envenoming by the viper [10]. The venom's phospholipases $A_{2}$, hemorrhagins, and procoagulant enzymes, including Factors $\mathrm{X}$ and $\mathrm{V}$ protease activators, are presumed to be responsible for these clinical manifestations $[10,12]$. However, striking variations in the clinical symptoms of envenomed victims [12,13] appeared to reflect some geographical differences in venom components [13].

Relative to other regions, Daboia snakebites in Myanmar and eastern India are known to cause severe internal bleeding and higher mortality $[14,15]$. Although the hemorrhagic protease VRR-73 was isolated from D. russelii, its properties and contribution to hemorrhage have not been clarified [16]. In the present study, we purified and characterized the corresponding hemorrhagins (namely daborhagin) of Daboia venoms from both regions and solved its full sequence. We also examined the occurrences of P-IIIs in the venoms from various true-viper species or subspecies, and did phylogenetic analyses to trace the evolution of daborhagin and other P-III SVMPs.

\section{Materials and methods}

\subsection{Venom and reagents}

Daboia venoms from Myanmar and eastern India were gifts from Professors Yu-Yen Shu and Antony Gomes [17]. Three individual $D$. siamensis venom samples from southern Myanmar were kindly given by Professor R. David G. Theakston. Venoms of Daboia russelii pulchella (Sri Lanka), D. russelii (Pakistan), D. siamensis (Taiwan), Echis leucogaster, Echis sochureki, Echis pyramidium, Vipera lebetina turanica, Vipera lebetina mauritanica, and Vipera ammodytes montandoni were purchased from Latoxan (Rosans, France). Venom of D. siamensis (Thailand) was from the Thailand Red-Cross, Bangkok, and venom of D. siamensis (Indonesia) was from Venom Supplies (Adelaide, Australia). Vipera ammodytes and Vipera berus venoms were from Sigma-Aldrich Co. (St Louis, MO, USA). Western Indian D. russelii venom was obtained from the Haffkine Institute (Mumbai, India). Other venoms used were from the Miami Serpentarium Laboratory (Punta Gorda, FL, USA).

Peptide: $N$ glycosidase F (PNGase F) and Endo $\mathrm{H}$ were from New England Biolabs (Beverly, MA, USA). Modification enzymes, restriction enzymes, broad-range protein markers, sequencing-grade modified trypsin, and pGEM-T vector were purchased from Promega Corp. (Madison, WI, USA). Protein substrates, insulin chain B, and other chemicals were from either Sigma-Aldrich or Merck (Darmstadt, Germany). Three fluorogenic substrates, (7-Methoxycoumarin-4-yl)acetylPro-Leu-Gly-Leu-(2,4-dinitrophenyl)-Ala-Arg- $\mathrm{NH}_{2}$ (FS-1), (7-Methoxycoumarin-4-yl)acetyl-Arg-Pro-Lys- Pro-Tyr-AlaNva-Trp-Met-Lys- $\left\{N^{\beta}-3\right.$-(2,4-dinitrophenyl)-L-2,3-diaminopropionyl $\}-\mathrm{NH}_{2}$ (NFF-2), and (7-Methoxycoumarin-4-yl)acetyl-Arg-Pro-Lys-Pro-Val-Glu-Nva-Trp-Arg-Lys- $\left\{N^{\beta}-3-(2,4-d i-\right.$ nitrophenyl)-L-2,3-diaminopropionyl $\}-\mathrm{NH}_{2}$ (NFF-3), were from the Peptide Institute Inc. (Osaka, Japan).

\subsection{Purification of P-III SVMPs}

Approximately $20 \mathrm{mg}$ of the crude venoms of $D$. siamensis (Myanmar) or D. russelii (Kolkata) were dissolved in $200 \mu \mathrm{l}$ of $0.1 \mathrm{M}$ ammonium acetate $(\mathrm{pH}$ 6.7) and then centrifuged at $12,000 \times g$ for $5 \mathrm{~min}$. The supernatant was then loaded to a Superdex G-75 column (10/300 GL; Pharmacia, Uppsala, Sweden) on an FPLC apparatus. The column was eluted at a flow rate of $1.0 \mathrm{ml} / \mathrm{min}$, and fractions of $0.5 \mathrm{ml}$ were collected. Fractions showing caseinolytic activities based on a colorimetrical assay [18] were collected. Pooled sample was lyophilized, redissolved, injected into a Mono Q column (5/ $50 \mathrm{GL}$; Pharmacia) pre-equilibrated with $50 \mathrm{mM}$ Tris- $\mathrm{HCl}$ buffer ( $\mathrm{pH} 8.0$ ), and then eluted with a $0-0.6 \mathrm{M} \mathrm{NaCl}$ gradient in the same buffer. Finally, the sample was purified using a Mono S column (HR 16/10; Pharmacia) pre-equilibrated with $10 \mathrm{mM}$ sodium phosphate $(\mathrm{pH}$ 6.7) and eluted with a $0-0.3 \mathrm{M} \mathrm{NaCl}$ gradient. Likewise, another two other P-IIIs were purified from $C$. vipera and E. leucogaster venoms, and designated as CVHRa $(99 \mathrm{kDa})$ and ECLV-DM $(97 \mathrm{kDa})$, respectively. The other three P-IIIs, Acurhagin, BHRa, and TSV-DM, were also purified according to published methods [18-20]. Their purity and molecular mass were confirmed by SDS-PAGE.

\subsection{Protein quantification}

Soluble crude venom and purified proteins were quantified using a BCA protein assay kit (Pierce Chemical Co., Rockford, IL, USA) with bovine serum albumin as a standard.

\subsection{Hemorrhage test}

The hemorrhagic activity of samples was measured by the published methods [21] with minor modifications. ICR mice (28-30 g) were shaved on their dorsal regions and then subcutaneously injected with samples in $100 \mu \mathrm{l}$ of PBS buffer. After $24 \mathrm{~h}$, the mice were sacrificed with an overdose of $\mathrm{CO}_{2}$, and the hemorrhagic spots were measured from the inside of the moved skins. The minimal hemorrhagic dose (MHD), defined as the amount of toxins causing a $10 \mathrm{~mm}$ skin-lesion in $24 \mathrm{~h}$, was determined from a plot of lesion diameters against the doses of toxins injected. Experiments involving mice and rabbits were done under the approval and followed the guidelines 
of the Animal Experiment Review Committee of the Academia Sinica, Taiwan.

\subsection{Mass spectrometry}

The precise molecular weight of daborhagin-M was analyzed in linear mode using the MALDI-TOF mass spectrometer (4700 Proteomics Analyzer; Applied Biosystems, Foster City, CA, USA) equipped with an Nd:YAG laser (355-nm wavelength and $200-\mathrm{Hz}$ repetition rate). One thousand shots were accumulated in positive ion mode. The sample was dissolved in $50 \%$ acetonitrile with $0.1 \%$ formic acid and premixed with a $5 \mathrm{mg} / \mathrm{ml}$ matrix solution of sinapic acid in $70 \%$ acetonitrile with $0.1 \%$ formic acid for spotting onto the target plate.

\subsection{Caseinolytic activity assay and inhibitor study}

Caseinolytic activity was measured colorimetrically as previously described [18]. The $100 \mu \mathrm{l}$ reaction mixture, containing $0.1 \mu \mathrm{g}$ of purified daborhagin, $0.5 \mathrm{mg}$ of azocasein in $50 \mathrm{mM}$ Tris- $\mathrm{HCl}$ buffer $\left(\mathrm{pH} 8.0\right.$ ), was incubated at $37^{\circ} \mathrm{C}$ for $90 \mathrm{~min}$. Reactions were quenched by adding $200 \mu \mathrm{l}$ of $5 \%$ trichloroacetic acid at room temperature. After spinning at $1000 \times g$ for $5 \mathrm{~min}, 150 \mu \mathrm{l}$ of the supernatant was mixed with an equal volume of $0.5 \mathrm{M} \mathrm{NaOH}$ to halt the reaction, and absorbance was determined at $450 \mathrm{~nm}$. One unit of proteolytic activity was defined as the amount of enzyme required to cause a 1.0 increase in absorbance at $450 \mathrm{~nm}$ per minute. Specific activity was expressed as units/mg protein. For the inhibitor study, $5 \mathrm{mM}$ of metal ions, chelators, or protein inhibitors were added to each reaction, separately.

\subsection{Hydrolysis of native proteins and oligopeptide substrates}

Fibrinogen and basement membrane proteins (collagen, laminin, and fibronectin) were incubated with daborhagin-M in $100 \mathrm{mM}$ Tris $-\mathrm{HCl}$ buffer $(\mathrm{pH} 8.0)$ at $37^{\circ} \mathrm{C}$. The reactions were terminated by adding reducing buffer, and boiled at $95{ }^{\circ} \mathrm{C}$ for $5 \mathrm{~min}$. The hydrolyzed products were then subjected to SDS-PAGE. The gels were stained with Coomassie Brilliant blue G-250 (Gelcode Blue Stain; Pierce Chemical).

Oxidized insulin chain-B $(715 \mu \mathrm{M})$ was incubated with $1.0 \mu \mathrm{M}$ daborhagin-M at $37^{\circ} \mathrm{C}$ in $50 \mathrm{mM}$ Tris buffer $(\mathrm{pH}$ 8.0). At various times, the reaction was halted by adding $25 \mathrm{mM}$ EDTA. Peptide products were separated using reverse phase HPLC on a Vydac $\mathrm{C}_{18}$-column, and monitored with the absorbance at $214 \mathrm{~nm}$. The peak fractions were collected and dried in a vacuum-centrifuge device (Labconco Corp., Kansas City, MO, USA). Each sample was analyzed using MALDITOF or electrospray ionization (ESI) mass spectrometry.

Stock solutions of synthetic fluorogenic substrates (FS-1, NFF-2, NFF-3) [22,23] were prepared in dimethyl sulfoxide. The P-III enzymes used for the kinetic study were freshly purified and kept active; their concentrations were determined based on their molecular weights. The assay was done by incubating a 1-ml mixture containing $10 \mathrm{nM}$ enzymes and $1 \mu \mathrm{M}$ substrate in $0.1 \mathrm{M}$ Tris $-\mathrm{HCl}(\mathrm{pH} 7.5)$ with $0.1 \mathrm{M}$ $\mathrm{NaCl}, 10 \mathrm{mM} \mathrm{CaCl}_{2}$, and $0.05 \%$ (w/v) Brij35. The increase in fluorescence intensity, in relative fluorescence units (RFU), was measured on a fluorescence spectrophotometer (F-3010; Hitachi Koki Co. Ltd., Tokyo, Japan), with excitation at $325 \mathrm{~nm}$ and emission at $393 \mathrm{~nm}$. The first-order rate constant obtained in each experiment was divided by the enzyme concentration to calculate its specificity index: $k_{\mathrm{cat}} / K_{\mathrm{m}}$.

The individual kinetic parameters, $K_{\mathrm{m}}$ and $k_{\mathrm{cat}}$, for the NFF2 substrate were also determined by analyzing experimental data with Lineweaver-Burk plots. The assay mixture contained 10-20 nM P-III enzymes and the concentration of the substrate was $0.2-5.0 \mu \mathrm{M}$.

\subsection{Antiserum and immunoblotting}

Approximately $250 \mu \mathrm{g}$ of purified daborhagin-M in phosphate-buffered saline was thoroughly mixed with an equal volume of Freund's complete adjuvant for the first injection, or Freund's incomplete adjuvant for the second and third injections. It was subcutaneously injected every two weeks into the back of a male rabbit biweekly. Ten days after the third injection, blood was taken from the rabbit's ear vein. The serum was harvested using centrifugation after it had clotted and been stored at $4{ }^{\circ} \mathrm{C}$. For Western blotting, $100 \mathrm{ng}$ of crude venoms was separated using $8 \%$ SDS-PAGE under non-reducing conditions. After the samples had been blotted onto a PVDF membrane, they were probed using anti-daborhagin-M antiserum (1:1000 dilution) and horseradish peroxidase-conjugated second antibody (1:2000 dilution). Immunoreactive bands were detected using the $\mathrm{NiCl}_{2}$ enhancement method [24].

\subsection{N-terminal sequencing}

To determine their $N$-terminal sequences, purified daborhagins $(10-20 \mu \mathrm{g} /$ well) were electrophoresed on a $1.0 \mathrm{~mm}$ thick $8 \%$ SDS-PAGE under reducing condition. The protein bands were blotted to a PVDF membrane. After the samples had been stained with Amido Black (0.2\% in $7 \%$ acetic acid), the bands were cut out and sequenced using a gas-phase amino acid sequencer (Procise 492; Applied Biosystems).

\subsection{Cloning and sequencing}

RNA and cDNA were prepared from a pair of venom glands obtained from a D. russelii specimen from eastern India [17]. PCR amplification of the daborhagin-K cDNA was done using specific primers: a sense degenerate 17-mer complementing the $N$-terminal residues $7-12$ (NRYFNP), and an antisense 18-mer designed according to the conserved sequences of far $3^{\prime}$-UTR of SVMPs [25]. To clone the $5^{\prime}$ upstream region, one primer based on the $5^{\prime}$-end conserved sequences and the other based on the amino acid sequence "AIDLNGL" of the protease domain were used. PCR conditions were as follows: initial denaturation for $2 \mathrm{~min}$ at $94{ }^{\circ} \mathrm{C}$, followed by 35 extension cycles (denaturation for $1 \mathrm{~min}$ at $94{ }^{\circ} \mathrm{C}$, annealing for 
$1 \mathrm{~min}$ at $52{ }^{\circ} \mathrm{C}$, and elongation for $1 \mathrm{~min}$ at $72{ }^{\circ} \mathrm{C}$ ), and a terminal extension step of $72{ }^{\circ} \mathrm{C}$ for $10 \mathrm{~min}$. The products were cloned into a pGEM-T vector, and the plasmids were transformed to Escherichia coli strain JM 109. White transformants were selected and positive clones were subjected to DNA sequencing using Taq-Dye-Deoxy terminator cycle sequencing kit (Applied Biosystems).

\subsection{Peptide mass fingerprinting}

For peptide mass fingerprinting (PMF), gel bands of native and deglycosylated daborhagins were excised separately and cut into pieces. Each sample was dehydrated with acetonitrile for $10 \mathrm{~min}$, dried, and then dissolved in $25 \mathrm{mM} \mathrm{NH}_{4} \mathrm{HCO}_{3}(\mathrm{pH}$ 8.5) containing $100 \mathrm{mM}$ dithioerythritol at $37^{\circ} \mathrm{C}$ for $1 \mathrm{~h}$. Its Cys-residues were alkylated with $65 \mathrm{mM}$ iodoacetamide at $27^{\circ} \mathrm{C}$ for $1 \mathrm{~h}$ in the dark. The protein was washed twice with $50 \%$ acetonitrile, dried, and hydrolyzed with $25 \mathrm{ng}$ of modified trypsin (Promega) in $25 \mathrm{mM} \mathrm{NH}_{4} \mathrm{HCO}_{3}(\mathrm{pH} \mathrm{8.5)}$ ) at $37{ }^{\circ} \mathrm{C}$ for $16 \mathrm{~h}$. The digest was twice extracted with $50 \%$ acetonitrile (containing 5\% formic acid) for $15 \mathrm{~min}$ each and then dried. The resultant peptides were analyzed using MALDITOF/TOF with a detecting mass range of 800-4000 Da.

\subsection{Phylogenetic analysis}

Amino acid sequences of 26 P-III SVMPs were retrieved using BlastP search. Their sequences were aligned using the Vector NTI program (Invitrogen Corp., Carlsbad, CA, USA). A phylogenetic tree was generated using the neighbor-joining methodology of the PHYLIP program, with an RVV-X heavy chain as an out-group. The degree of confidence was determined using bootstrap analyses of 1000 replicates [26].

\subsection{Absolute complexity plot and homologous modeling}

The absolute complexity plot shows the average of pairwise alignment scores of each residue using the substitution matrix blosum62mt2. Individual plots were generated using the alignment of the members from each class with the AlignX module of the Vector NTI program.

The 3D-model of daborhagin was built using the Modeller program (http://salilab.org/modeller), with the crystal structure of catrocollastatin/VAP2B (PDB code 2DW0) from Crotalus atrox venom [27] as a template. Model geometry was analyzed using the PROCHECK program. The ribbon diagrams were generated using the PyMOL program (http://pymol.sourceforge.net/).

\section{Results}

\subsection{Purification and characterization of daborhagin}

The crude venom of $D$. siamensis (Myanmar) was separated into several fractions using a Superdex G-75 column (Fig. 1A). The first fraction, which showed the strongest caseinolytic
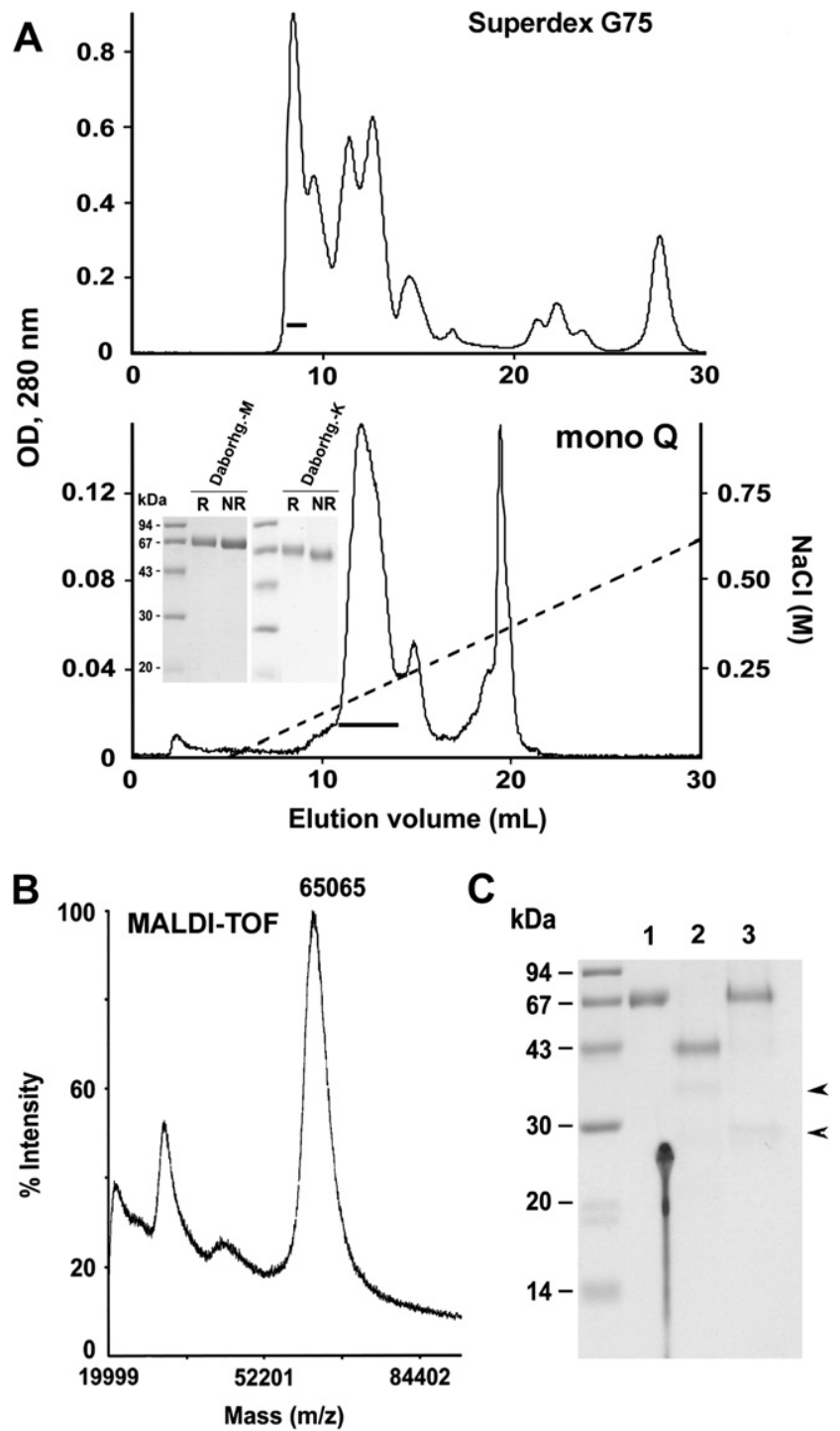

Fig. 1. Purification and characterization of daborhagins. (A) Myanmar Daboia siamensis venom was separated using a Superdex G-75 column equilibrated with $0.1 \mathrm{M}$ ammonium acetate $(\mathrm{pH}$ 6.7), and then a Mono $\mathrm{Q}$ column with a $0-0.6 \mathrm{M} \mathrm{NaCl}$ gradient in $50 \mathrm{mM}$ Tris $-\mathrm{HCl}(\mathrm{pH} 8.0)$. The active fractions are indicated by bars. The inset indicates the SDS-PAGE patterns of purified daborhagin-M and $-\mathrm{K}$ under reducing $(\mathrm{R})$ and non-reducing $(\mathrm{NR})$ conditions. (B) The precise molecular weight of native daborhagin-M was analyzed using MALDI-TOF. (C) Daborhagin-M $(4 \mu \mathrm{g})$ was incubated with buffer only (lane 1) or with 1 unit of PNGase F (lane 2) or 5 units of Endo $\mathrm{H}$ (lane 3) at $37^{\circ} \mathrm{C}$ for $3 \mathrm{~h}$. Samples were then subjected to SDS-PAGE under reducing conditions. Arrowheads indicate deglycosylation enzymes.

activity, was collected. After it had been desalted and concentrated, it was further partitioned using ion-exchange chromatography on Mono Q (Fig. 1A) and Mono S (data not shown) columns. The purified active component induced hemorrhage with a minimum hemorrhagic dose (MHD) of $0.86 \mu \mathrm{g}$ when subcutaneously injected into mice. Based on the species origin (Dabo-), hemorrhagic activity (-rhagin), and geographic region (Myanmar), we designated it daborhagin-M. Using the same procedures, we purified another hemorrhagin from the venom of D. russelii (Kolkata, eastern India) and designated it daborhagin-K. Its hemorrhagic potency with a MHD of $0.82 \mu \mathrm{g}$ 
was similar to that of daborhagin-M. The amounts of daborhagin- $\mathrm{M}$ and daborhagin- $\mathrm{K}$ in the crude venoms were estimated to be $5.5 \%$ and $0.8 \%(\mathrm{w} / \mathrm{w})$, and their specific activities toward azocasein were 7.1 and 7.3 units/mg, respectively.

SDS-PAGE analysis of both daborhagins revealed a single band with an apparent mass of 62 and $66 \mathrm{kDa}$ under non-reducing and reducing conditions, respectively (Fig. 1A, inset). We further determined the mass of daborhagin-M using MALDI-TOF mass spectrometry. We identified a singlecharged monomer with an average molecular mass of $65,065 \mathrm{Da}$ and a double-charged monomer $(\mathrm{m} / \mathrm{z} 31,627)$ (Fig. 1B). PNGase F treatment reduced the mass to $43 \mathrm{kDa}$, but Endo $\mathrm{H}$ treatment did not affect it (Fig. 1C), which suggested that daborhagin-M contains $4 \sim 5$ complex type $N$ linked glycans. To examine its general proteolytic activities, we used various divalent metal ions and proteinase inhibitors in regular azocasein assays (data not shown). Adding $5 \mathrm{mM}$ of $\mathrm{Ca}^{2+}$ or $\mathrm{Mg}^{2+}$ increased the caseinolytic activities of the purified daborhagin-M by $5-23 \%$, and adding $5 \mathrm{mM}$ of EDTA, EGTA, or 1,10-phenanthroline strongly inhibited its activities by $84-94 \%$. However, serine protease inhibitor, e.g. PMSF, was not inhibitory. These results suggested that daborhagin-M is a high molecular weight metalloproteinase.

\subsection{Proteolytic activity toward plasma and basement membrane proteins}

The specificities of daborhagin-M were studied using potential plasma and matrix proteins as substrates. At a low enzyme concentration of $75 \mathrm{nM}$, daborhagin-M cleaved the A $\alpha$-chain of human fibrinogen specifically within minutes, but it had no apparent effect on B $\beta$ - and $\gamma$-chains (Fig. 2A). Thus, daborhagin-M is a new $\alpha$-fibrinogenase. Notably, it completely degraded high molecular weight subunits $(>200 \mathrm{kDa})$ of type IV collagen in $2 \mathrm{~h}$, and partially degraded a $250 \mathrm{kDa}$ main chain of fibronectin into five fragments of $83-225 \mathrm{kDa}$. By contrast, we barely detected laminin hydrolysis after $24 \mathrm{~h}$ (data not shown).

\subsection{Cleavage sites on insulin chain-B}

We also used oxidized insulin chain-B to examine the proteolytic specificity of daborhagin (Fig. 2B). After oxidized insulin chain-B had been hydrolyzed by $1.0 \mu \mathrm{M}$ daborhagin-M at $37^{\circ} \mathrm{C}$, its products at various incubation times $(10 \mathrm{~min}-$ $24 \mathrm{~h}$ ) were isolated using reverse-phase HPLC, and then each purified oligopeptide was analyzed using MALDI-MS spectrometry (data not shown). We found that daborhagin-M had cleaved chain-B at four sites (e.g. X-Leu and X-Phe), similar to other SVMPs [28]. The fast cleavages were at Ala ${ }^{14}$ $\mathrm{Leu}^{15}$ and $\mathrm{Tyr}^{16}-\mathrm{Leu}^{17}$, followed by those at His ${ }^{10}-\mathrm{Leu}^{11}$, and the slow cleavages were at $\mathrm{Phe}^{24}-\mathrm{Phe}^{25}$ (Fig. 2B).

\subsection{Kinetic study using fluorogenic substrates}

The fluorogenic peptide substrates FS-1 (cleaving at GlyLeu), NFF-2 (cleaving at Ala-Nva), and NFF-3 (cleaving at Glu-Nva) were originally developed to measure matrix metalloproteinase (MMP) activity [22,23]. To study the relationship between substrate specificity and hemorrhagic potency, we compared the kinetic specificities of daborhagin-M and the other five P-IIIs toward these substrates. The specificity index $\left(k_{\mathrm{cat}} / K_{\mathrm{m}}\right)$ of daborhagin-M toward NFF-2 was about 3-4 times higher than those toward FS-1 or NFF-3 (Table 1). Other strong venom hemorrhagins-BHRa of Bitis arietans venom [19], CVHRa of C. vipera venom, and acurhagin of Deinagkistrodon acutus venom [18]-also showed higher $k_{\mathrm{cat}} / K_{\mathrm{m}}$ values toward NFF-2 or NFF-3 than the weak hemorrhagins, e.g. TSV-DM of Trimeresurus stejnegeri venom [20] and ECLV-
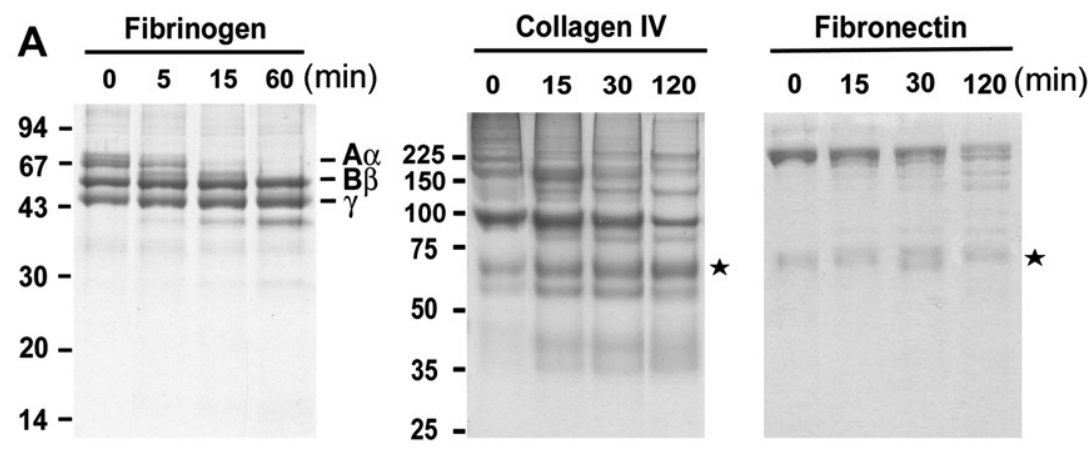

B

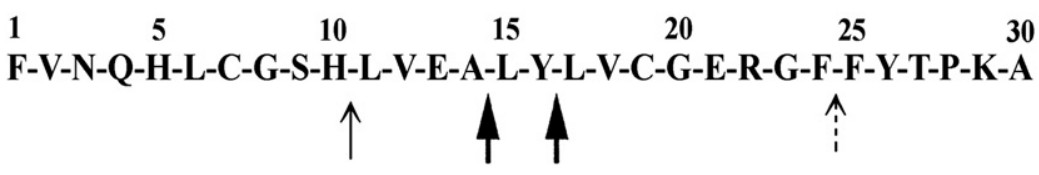

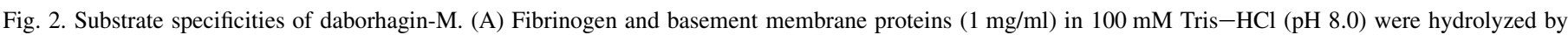

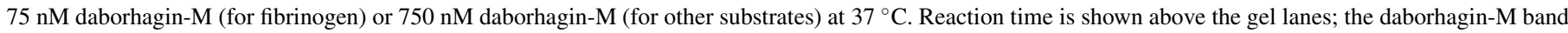

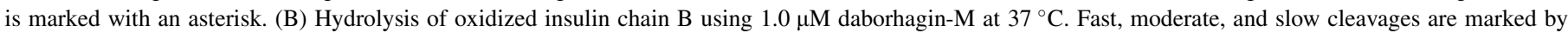
thick arrows, thin solid-line arrow and thin dashed-line arrow, respectively. 
Table 1

Kinetic specificities of P-III SVMPs toward three synthetic oligopeptides in $0.1 \mathrm{M}$ Tris- $\mathrm{HCl}(\mathrm{pH} 7.5)$ at $25^{\circ} \mathrm{C}$

\begin{tabular}{llrrrrr}
\hline Class & P-III enzymes & \multicolumn{2}{c}{$k_{\text {cat }} / K_{\mathrm{m}} \times 10^{-3}, \mathrm{M}^{-1} \mathrm{~s}^{-1}$} & $\begin{array}{l}k_{\text {cat }} \\
\left(\mathrm{s}^{-1}\right)\end{array}$ & $\begin{array}{l}K_{\mathrm{m}} \\
\left(\mu \mathrm{M}^{-1}\right)\end{array}$ \\
\cline { 3 - 7 } & & $\mathrm{FS}-1$ & $\mathrm{NFF}-3$ & $\mathrm{NFF}-2$ & $\mathrm{NFF}-2$ & NFF-2 \\
\hline $\mathrm{HH}$ & Daborhagin-M & 30.6 & 41.2 & 125.0 & 0.88 & 7.0 \\
& BHRa & 3.2 & 128.7 & 23.7 & 0.13 & 5.5 \\
& CVHRa & 11.0 & 38.4 & 96.4 & 1.26 & 13.0 \\
& Acurhagin & 18.2 & 41.9 & 34.2 & 0.32 & 9.4 \\
\multirow{2}{*}{ NH } & ECLV-DM & 0.4 & 3.8 & 3.2 & 0.05 & 16.1 \\
& TSV-DM & 1.9 & 46.1 & 1.2 & 0.03 & 25.0 \\
& & & & & & \\
\multirow{2}{*}{ Other } & RVV-X & 0.2 & 5.7 & 0.2 & $-{ }^{\mathrm{a}}$ & - \\
\hline
\end{tabular}

Substrate structures are shown in Section 2.

a - , not determined.

DM of E. leucogaster venom (Table 1). By contrast, RVV-X barely hydrolyzed these synthetic substrates.

We also determined the $K_{\mathrm{m}}$ and $k_{\text {cat }}$ values of all the above P-IIIs for substrate NFF-2. The data suggested that lower $K_{\mathrm{m}}$ and much higher $k_{\text {cat }}$ values accounted for the faster hydrolysis of NFF-2 by the strong hemorrhagic P-IIIs than the weak or non-hemorrhagic P-IIIs (Table 1).

\subsection{Occurrence of daborhagin-like enzymes in Daboia and other viperid venoms}

Anti-daborhagin antiserum was prepared by immunizing one rabbit with native daborhagin-M; the antiserum reacted similarly to both daborhagins and easily recognized them below $1.0 \mathrm{ng}$ in Western blot analysis (Fig. 3A). To avoid falsepositive results, only $100 \mathrm{ng}$ of each venom sample was loaded into the gel. Daborhagins were easily detected in Myanmar and eastern India Daboia venoms, with estimated levels of $8.0 \%$ and $1.5 \%$ (w/w of total soluble venom proteins) (Fig. 3B), respectively. A faint $60-\mathrm{kDa}$ band was also detected in western India D. russelii venom. However, no antigen was detected in the Daboia venoms collected from Pakistan, Sri Lanka, Thailand, Indonesia, and Taiwan. Thus, daborhagin is found in Daboia venoms only in particular geographical regions. Western blotting also revealed that three of the Daboia venom samples collected from southern Myanmar contained abundant daborhagins (data not shown).

To further explore the distribution of similar P-III enzymes in other viperid venoms, ten available venom samples under the genera Vipera, Cerastes, and Echis were also tested. The results suggested that various P-III enzymes are simultaneously present in most viperid venoms (Fig. 3C). Notably, $C$. vipera venom is especially rich in P-III enzymes, whereas European viper venoms such as $V$. ammodytes and $V$. berus had low P-III levels.

\section{6. cDNA cloning and the predicted sequence of daborhagin-K}

The cDNA of daborhagin-K was cloned and sequenced. The open reading frame of the daborhagin-K precursor
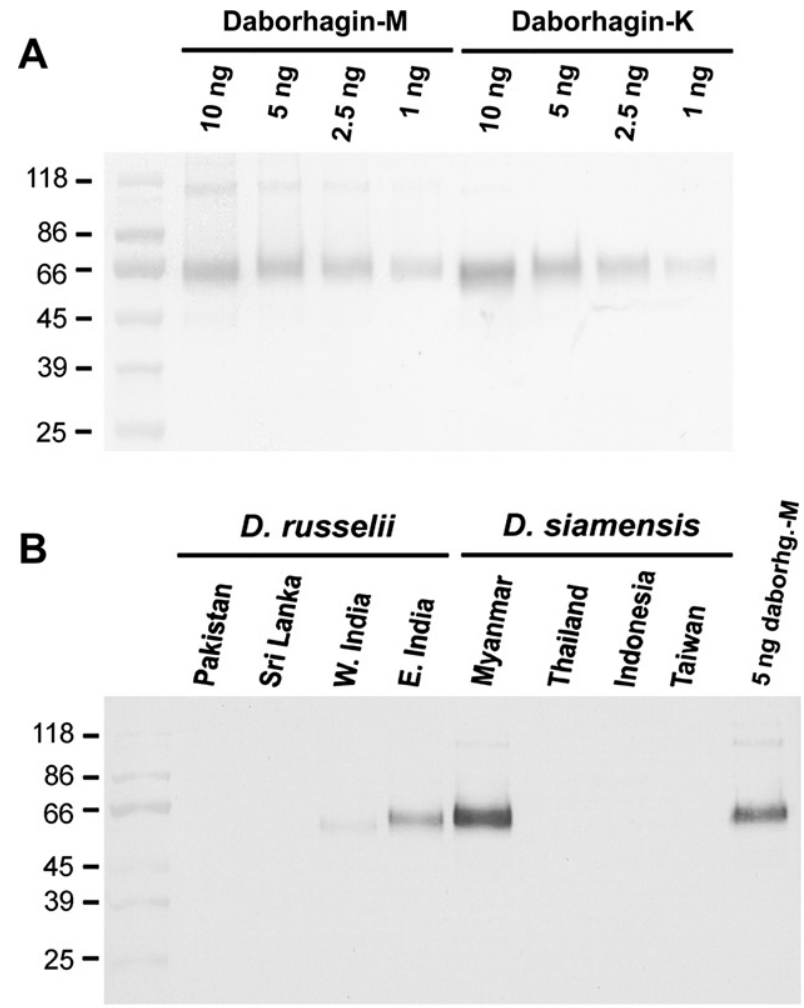

C

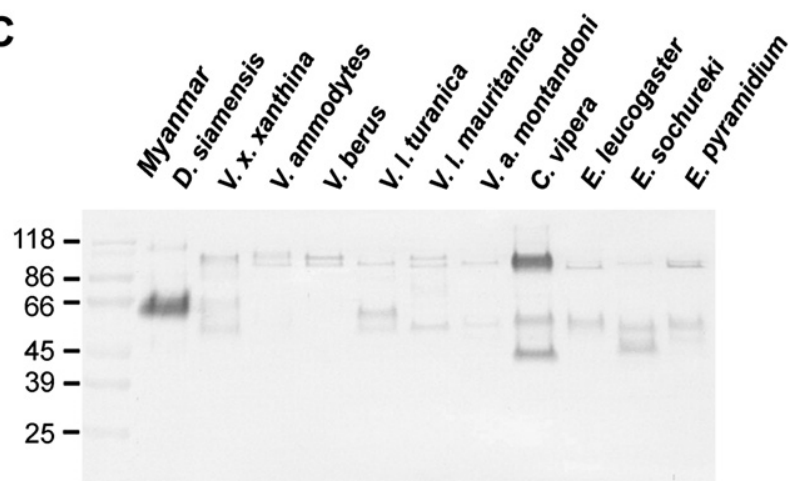

Fig. 3. Detection of daborhagin-like or P-III SVMPs in Daboia and other viperid venoms by Western blot analysis. (A) Serological cross-reactivity of both daborhagins with anti-daborhagin-M polyclonal antibodies. (B) Analysis of eight Daboia geographical samples. W. India and E. India denote the venoms from Mumbai and Kolkata, respectively. (C) Analysis of ten venom samples from other Viperinae species. In (B) and (C), each sample containing $100 \mathrm{ng}$ of soluble crude venom proteins was separated using SDS-PAGE $(8 \%)$ under non-reducing conditions. After blocking, the samples on blotting membranes were probed with anti-daborhagin-M polyclonal antibodies.

encoded 615 amino acid residues, including a highly conserved 18-residue signal peptide and a 171-residue proenzyme domain (data not shown). $N$-terminal sequences of predicted mature enzyme nicely matched that of purified daborhagin$\mathrm{K}$, which were determined as VATSERNRYFNPYSYV by automatic sequencer. The deduced daborhagin-K contained 426 residues (with a calculated molecular mass of 48,041 Da) including three classic structural domains of P-IIIs (Fig. 5). Additionally, four potential $N$-glycosylation sites at Asn 74, 80, 189, and 339 were found, which accorded with our observation of a $17-\mathrm{kDa}$ mass increase due to glycosylation (Fig. 1C). 
To verify the sequence, native and deglycosylated daborhagin-K were digested in gel with trypsin, and the resulting peptides were analyzed using MALDI-TOF/TOF. The resultant 23 peptide fragments matched those predicted, and they represented more than $70 \%$ coverage of the entire sequence (Table 2). Similarly, 11 peptide fragments of daborhagin-M also matched those predicted from the digestion of daborhagin-K. Thus, daborhagin- $\mathrm{K}$ and $-\mathrm{M}$ shared a high degree of identity in their primary structures.

\subsection{Molecular phylogeny and classification of P-IIIs}

A phylogenetic tree was constructed based on 27 amino acid sequences of P-III SVMPs; some of them were predicted from cDNA clones and their functions have not been characterized. The topology of the tree reveals three major clusters of P-III enzymes (Fig. 4). Based on their MHD values (shown in parentheses), one cluster containing highly hemorrhagic PIII members is designated as the HH class, and another cluster containing mainly weakly or non-hemorrhagic enzymes is designated as the NH class. The other small cluster is comprised of prothrombin activators derived from Echis venom. Notably, daborhagin- $\mathrm{K}$ is associated with other strong hemorrhagins, e.g. HR1a (from Protobothrops flavoviridis) and HF3 (from Bothrops jararaca) within the $\mathrm{HH}$ class, but not with the PIIIa and P-IIIc members [2,9].

\subsection{Sequence alignment and comparison between the $\mathrm{HH}$ and $\mathrm{NH}$ classes}

Protein sequences of daborhagin-K and the other eight P-III enzymes selected from each lineage of the phylogeny tree were aligned (Fig. 5). Their $\mathrm{p} I$ values, potential $N$-glycosylation sites, numbers of Cys-residues, and classification subtypes [2,9] were also listed for comparison. Like other P-IIIs, daborhagin contains 17 intramolecular disulfide bonds; its zincchelating motif, Met-turn (CIM), and three $\mathrm{Ca}^{2+}$ binding sites [29] are highly conserved. In addition, its disintegrin domain contains a DECD sequence instead of the RGD or KGD found in P-II members [2]. Two hydrophobic ridges (HR) at positions 332-333 and 356-357, which possibly create a novel interaction surface with the hyper-variable region (HVR) at 383-410 [29], were also present.

To elucidate the molecular features responsible for distinguishing the $\mathrm{HH}$ and $\mathrm{NH}$ classes, we further constructed their individual absolute complexity profiles (Fig. 6A). By examining the superimposed topographies of both profiles, we found that most regions were similar. However, four structural elements (designated as M1, M2, C1, and C2) were found to bear significantly higher absolute complexity in $\mathrm{HH}$ than in $\mathrm{NH}$ members. The consensus sequences of M1, M2, C1, and $\mathrm{C} 2$ in $\mathrm{HH}$ members were ${ }^{137}$ YSPINLV $^{143},{ }^{176}$ PVISxxPSKF $^{186}$ (x represents a less-conserved residue), ${ }^{353} \mathrm{KGNY}^{356}$, and

Table 2

Match between the calculated $\left(\mathrm{MS}_{\mathrm{c}}\right)$ and the experimental $\left(\mathrm{MS}_{\mathrm{e}}\right)$ masses of tryptic fragments of daborhagins

\begin{tabular}{|c|c|c|c|c|}
\hline \multirow[t]{2}{*}{ Tryptic peptides of daborhagin-K } & \multirow[t]{2}{*}{ Position } & \multirow[t]{2}{*}{$\mathrm{MS}_{\mathrm{c}}{ }^{\mathrm{a}}$} & \multicolumn{2}{|l|}{$\mathrm{MS}_{\mathrm{e}}$} \\
\hline & & & Daborhagin-K & Daborhagin-M \\
\hline VATSERNR & $1-8$ & 932.49 & 932.47 & $-^{b}$ \\
\hline NRYFNPYSYVELIITVDHSMVTK & $7-29$ & 2805.39 & 2805.33 & - \\
\hline YFNPYSYVELIITVDHSMVTK & $9-29$ & 2535.25 & 2535.22 & - \\
\hline YKNDLTAIR & $30-38$ & 1093.60 & 1093.59 & 1093.60 \\
\hline IHDNSQLLTAIDLNGLTIGMAYVSTMCQSK & $99-128$ & 3326.60 & 3326.43 & - \\
\hline YSVGVVQDHSKINLR & $129-143$ & 1714.92 & 1714.90 & - \\
\hline YFSNCSYNQYR & $185-195$ & 1501.62 & 1501.75 & - \\
\hline YFSNCSYNQYRR & $185-196$ & 1657.72 & 1657.79 & - \\
\hline RFLTEHNPECIINPPLR & $196-212$ & 2106.09 & 2106.07 & 2106.06 \\
\hline FLTEHNPECIINPPLR & $197-212$ & 1949.99 & 1949.98 & 1949.99 \\
\hline TDIVSPPACGNELLER & $213-228$ & 1770.87 & 1770.86 & 1770.84 \\
\hline LHSWVECESGKCCNQCR & $252-268$ & 2209.90 & 2209.92 & 2209.92 \\
\hline RAGTECRPARDECDKAEQCTGR & $271-292$ & 2623.15 & 2623.17 & - \\
\hline AGTECRPARDECDKAEQCTGR & $272-292$ & 2467.05 & 2467.05 & 2467.06 \\
\hline SANCPVDEFHENGRPCLHNFGYCYNGK & $293-319$ & 3242.36 & 3242.23 & - \\
\hline CPIMYHQCHALFGQNVTGVQDSCFQYNR & $320-347$ & 3446.49 & 3446.32 & - \\
\hline LGVYYAYCR & $348-356$ & 1164.55 & 1164.54 & - \\
\hline LGVYYAYCRK & $348-357$ & 1292.65 & 1292.63 & - \\
\hline ENGRKIPCAPKDEK & $358-371$ & 1584.82 & 1584.67 & 1584.68 \\
\hline LYCSYKSPGNQIPCLPYYIPSDENK & $375-399$ & 3006.40 & - & 3006.36 \\
\hline CGRLYCSYK & $372-380$ & 1206.54 & 1206.58 & - \\
\hline SPGNQIPCLPYYIPSDENK & $381-399$ & 2192.03 & 2192.00 & 2192.00 \\
\hline SPGNQIPCLPYYIPSDENKGMVDHGT & $381-407$ & 3033.40 & 3033.27 & 3033.38 \\
\hline CGDGKVCSNGQCVDLNIAY & $408-426$ & 2129.90 & 2129.98 & 2129.93 \\
\hline
\end{tabular}

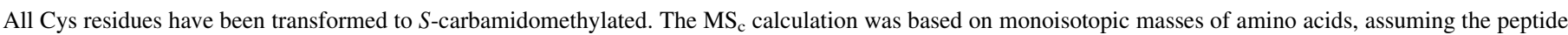
mass as $[\mathrm{M}+\mathrm{H}]^{+}$.

a The calculated masses $\left(\mathrm{MS}_{\mathrm{c}}\right.$ ) of tryptic peptides are based on predicted daborhagin-K sequence.

b - , not found in the experimental MS data. 


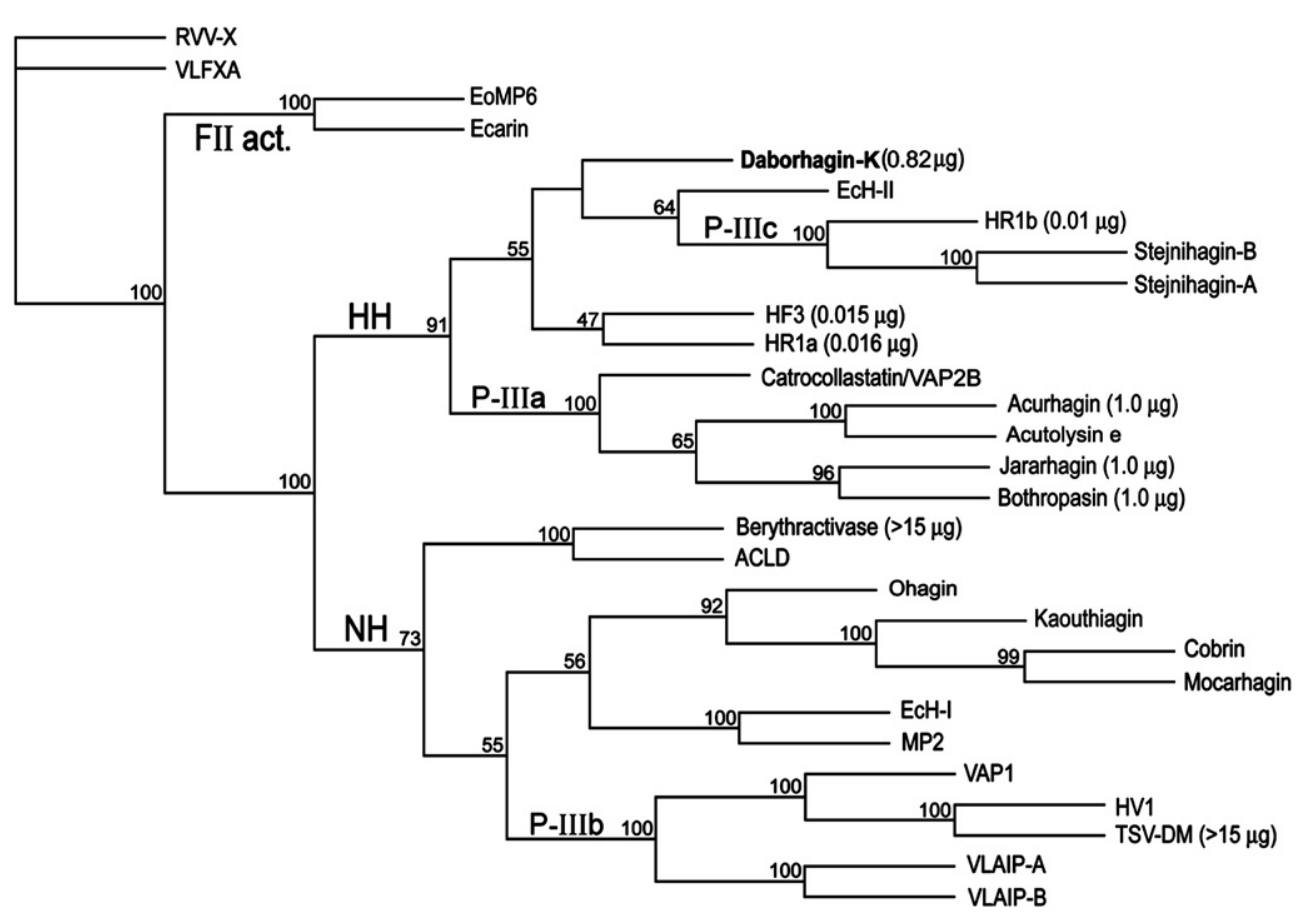

Fig. 4. Phylogenetic tree of P-III SVMPs based on amino acid sequences. The RVV-X heavy chain (AAB22477) was the out-group. Bootstrap values are shown at each node and MHD values are shown in parentheses. Abbreviations: FII act., prothrombin activators; HH, highly hemorrhagic class; NH, non-hemorrhagic class. The accession numbers and species are: Daborhagin-K (DQ137798), Daboia russelii; ACLD (AAC18911), Agkistrodon contortrix laticinctus; Acurhagin [18] (AAS57937) and Acutolysin e (AAD27891), Deinagkistrodon acutus; MP-2 (AAX86634), Bitis arietans; Bothropasin (AAC61986), HF3 [49] (AAG48931), and Jararhagin [50] (CAA48323), Bothrops jararaca; Berytractivase [6] (AAL47169), Bothrops erythromelas; Catrocollastatin/VAP2B [27] (AAC59672) and VAP1 [29] (BAB18307), Crotalus atrox; Ecarin [5] (Q90495), Echis carinatus; EoMP06 (AAP92424), Echis ocellatus; EcH-I (CAA55565) and EcH-II (CAA55566), Echis pyramidum leakeyi; Ohagin (ABM87941), Ophiophagus hannah; Kaouthiagin [51] (P82942), Naja kaouthia; Cobrin (AAF00693), Naja naja; Mocarhagin (AAM51550), Naja mossambica; HR1a (BAB92013), HR1b [52] (BAB92014), and HV1 (BAB60682), Protobothrops flavoviridis; TSV-DM [20] (ABC73079), Stejnihagin-A [9] (ABA40760), and Stejnihagin-B [9] (ABA40759), Trimeresurus stejnegeri; VLFXA HC (AAQ17467), VLAIP-A [53] (AAX38182), and VLAIP-B [53] (AAX38182), Macrovipera lebetina.

${ }^{401} \mathrm{KYFY}^{404}$ (Fig. 6B). The corresponding locations of these four regions are shown in the $3 \mathrm{D}$ model of daborhagin (Fig. 6C).

\section{Discussion}

To address the problem of highly hemorrhagic symptoms elicited by Myanmar and eastern India Daboia envenoming [13-15], we isolated the active P-III protease, daborhagin, from both Daboia venoms (Fig. 1). When subcutaneously injected into dorsal skin of mice, daborhagins caused severe hemorrhage with a MHD of $0.8-0.9 \mu \mathrm{g}$. Thus, daborhagins are highly hemorrhagic (MHD $<1 \mu \mathrm{g}$ ) toxins [30]. We also showed that daborhagin is a potent $\alpha$-fibrinogenase, similar to the VaH1 hemorrhagic P-III from $V$. ammodytes venom [31], but it has no effect on collagen- or ADP-induced platelet aggregation (data not shown). Daborhagin thus is able to interfere with the homeostatic system by degrading plasma fibrinogen. Furthermore, it has highly proteolytic activities toward type IV collagen and fibronectin, corroborating that the enzymatic hydrolysis of matrix components is a key event of SVMP-induced microvessel disruption [3,30,32]. A recent study [4] reported that jararhagin (P-III class) was more potently hemorrhagic than BaP1 (P-I class) because it selectively cleaved key peptide bonds in mouse nidogen. Therefore, daborhagin appears to be another good model for the biochemical study of hemorrhagic mechanisms induced by SVMPs.

Western blot analyses showed that only the Daboia venoms from Myanmar and eastern India have high levels of daborhagin (Fig. 3), and, indeed, that the venom of Daboia from Myanmar contains 6-7 times more daborhagin than that from eastern India. Such results are consistent with the fact that Daboia snakebites in both regions are particularly lethal and hemorrhagic, and that Myanmar's viper envenoming frequently causes gastrointestinal or respiratory tract bleeding as well as pituitary infarction [13-15]. Thus, daborhagins are most likely critical in these syndromes. Recently, we found that the $\mathrm{PLA}_{2}$ isoforms isolated from Daboia venom of both regions share $97-100 \%$ sequence identities [17]. All these venom similarities strongly support that these two Daboia populations are closely related and represent a special lineage of this genus, which may explain why Myanmar antivenom for Russell's viper was not effective against Thailand and Sri Lanka Russell's viper venoms [33,34]. This is useful information for Daboia antivenom production and the clinical management of Daboia envenomed victims.

Daborhagin-M and -K are orthologous genes in two Daboia species, but they have some microheterogeneities imbedded in their sequences. First, both enzymes were isolated from $\mathrm{Da}$ boia venoms using similar steps and had comparable 


\begin{abstract}
Metalloprotease domain

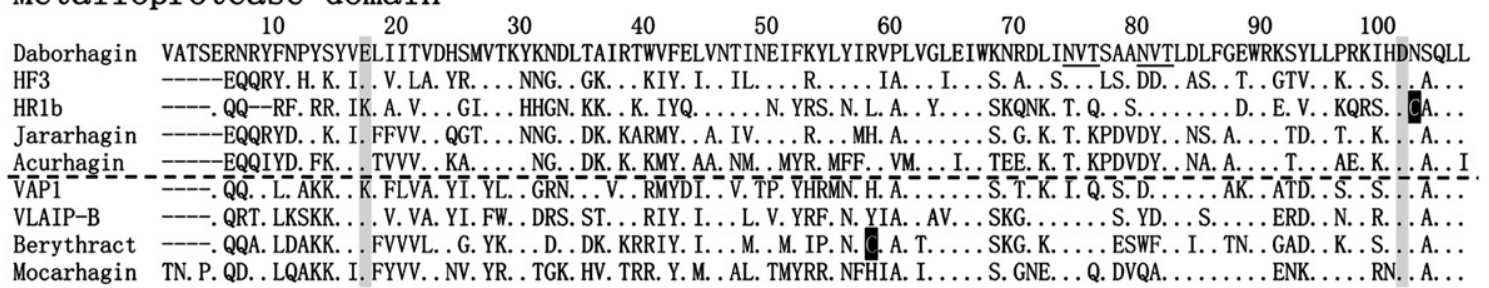

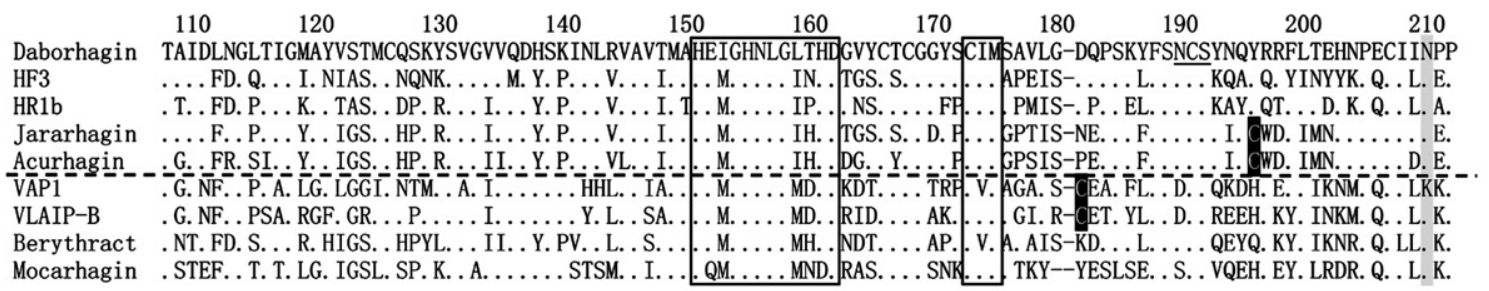

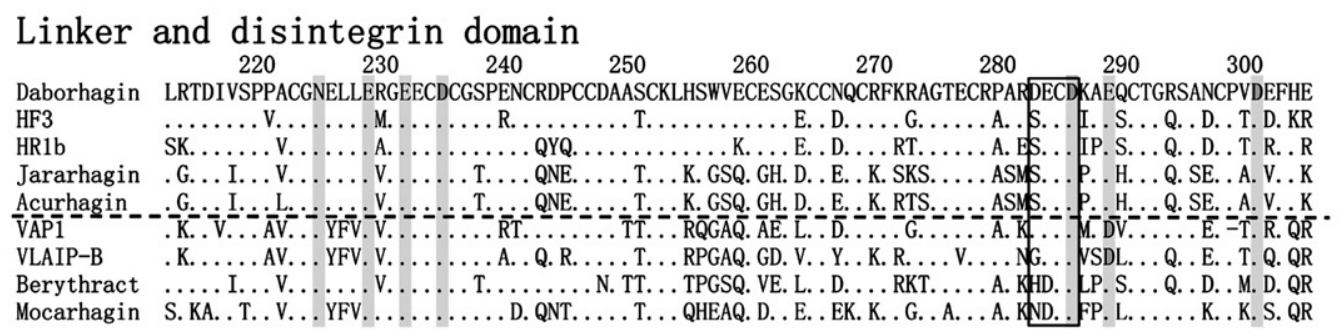

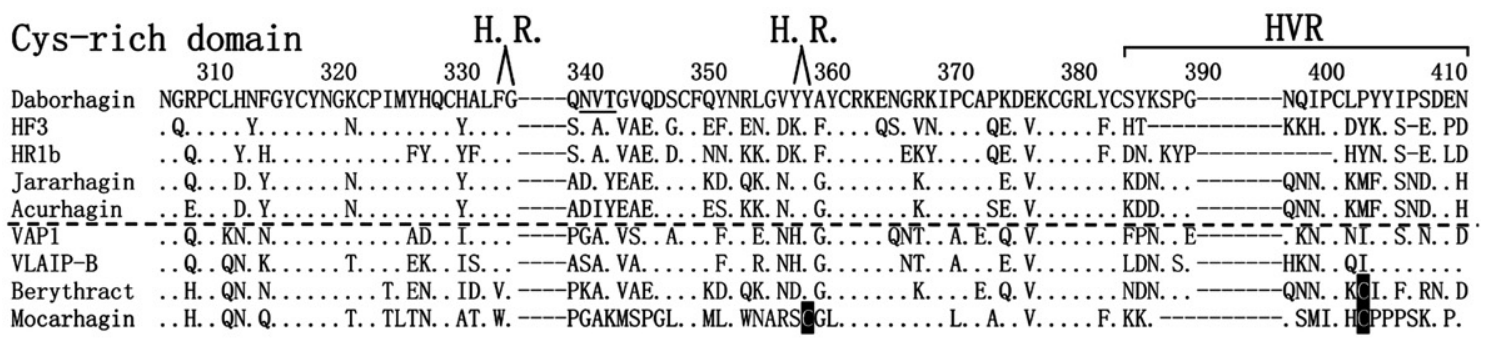

\begin{tabular}{|c|c|c|c|c|c|c|}
\hline & $\begin{array}{lll}420 & 430 & 441\end{array}$ & identity $(\%)$ & $\mathrm{pI}$ & No. of $N$-glycan & No. of Cys & Subclass \\
\hline Daborhagin & KGMVDHGTKCGDGKVCS-NGQCVDLNIAY-- & 100 & 6. 90 & 4 & 34 & \\
\hline & Y....N....A..... ${ }^{-} \ldots$ H. . VAT. . - & 63 & 5.26 & 5 & 34 & \\
\hline HR1b & 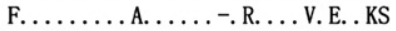 & 60 & 6.02 & 4 & 35 & PIIIc \\
\hline $\begin{array}{l}\text { Jararhagin } \\
\text { Acurhagin }\end{array}$ & $\begin{array}{l}-\ldots \text { H. . VAT. } \\
- \\
-\end{array}$ & 61 & $\begin{array}{l}5.07 \\
4.94\end{array}$ & 1 & $\begin{array}{l}35 \\
35\end{array}$ & $\begin{array}{l}\text { PIIIa } \\
\text { PIIIa }\end{array}$ \\
\hline VAP1 & LP. . A. R. A. $-\ldots \ldots$ VTTP. $=$ & 57 & 7. & 1 & -35 & PIÎ́b- \\
\hline VLAIP-B & 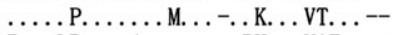 & 60 & 7.82 & 4 & 35 & PIIIb \\
\hline Berythract & R..LP....A. ... ${ }^{-}$RH. . VAT. .-- & 56 & 6.15 & 4 & 36 & \\
\hline Mocarhagin & Y...AP. ....VKK...R-. R. . KV------ & 50 & 8.77 & 2 & 36 & \\
\hline
\end{tabular}

Fig. 5. Alignment of amino acid sequences of daborhagin-K with those of representative HH and NH members. The HH sequences are above the dashed lines, and the NH sequences are below. Residues identical to those of daborhagin-K are denoted by dots, and the gaps are marked with hyphens. Potential $\mathrm{N}$-glycosylation sites of daborhagin-K are underlined. $\mathrm{Ca}^{2+}$-binding sites and non-conserved Cys-residues are shaded in gray and black, respectively. Conserved zinc-binding sites, Met-turns, and ECD motifs are boxed. Two hydrophobic ridges (HR) and hyper-variable regions (HVR) are also marked.

hemorrhagic potencies. Second, their masses were almost identical according to SDS-PAGE analysis. Third, anti-daborhagin- $\mathrm{M}$ antiserum easily detected daborhagin-K in Western blot analysis, which indicated that they are closely related and share common epitopes. Furthermore, tryptic PMF analysis of daborhagin-M and $-\mathrm{K}$ confirmed that they both had 11 and 23 unique peptides, which nicely matched those predicted from daborhagin-K cDNA (Table 2). Moreover, the $N$-terminal sequence $(1-25)$ of daborhagin-M is identical to that of daborhagin-K, except for the replacement of the $\operatorname{Pro}^{6}$ with $\mathrm{Arg}^{6}$.
Upon obtaining the full primary sequence of daborhagin-M, one may clarify how many heterogeneities exist between them.

The previously reported VRR-73 $(\mathrm{MHD}=0.5 \mu \mathrm{g})$ [16] showed comparable masses and hemorrhagic potencies with daborhagin-K, and both were derived from eastern India $D$. russelii venom. Like other hemorrhagic SVMPs, daborhagin$\mathrm{K}$ contains one typical zinc-chelating motif and three $\mathrm{Ca}^{2+}$ binding sites. Indeed, adding $5 \mathrm{mM} \mathrm{Mg}^{2+}$ or $\mathrm{Ca}^{2+}$ to daborhagin-K increased the casein hydrolysis rate by $13-23 \%$. 
A

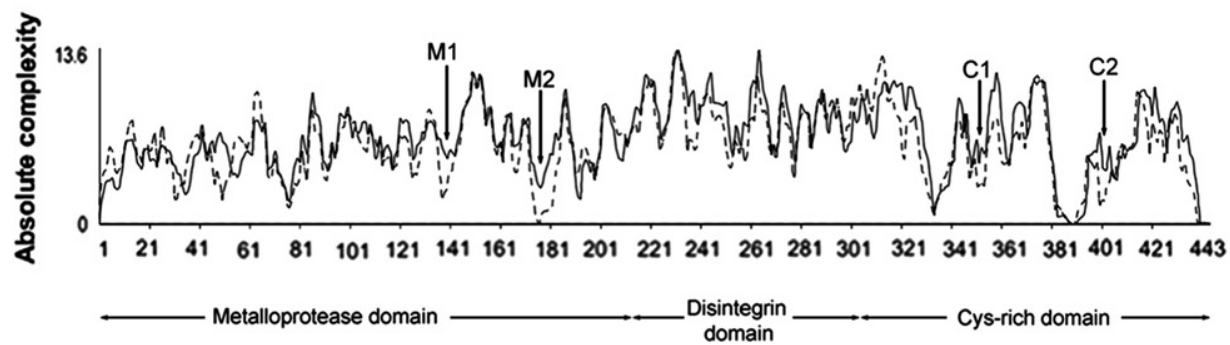

B
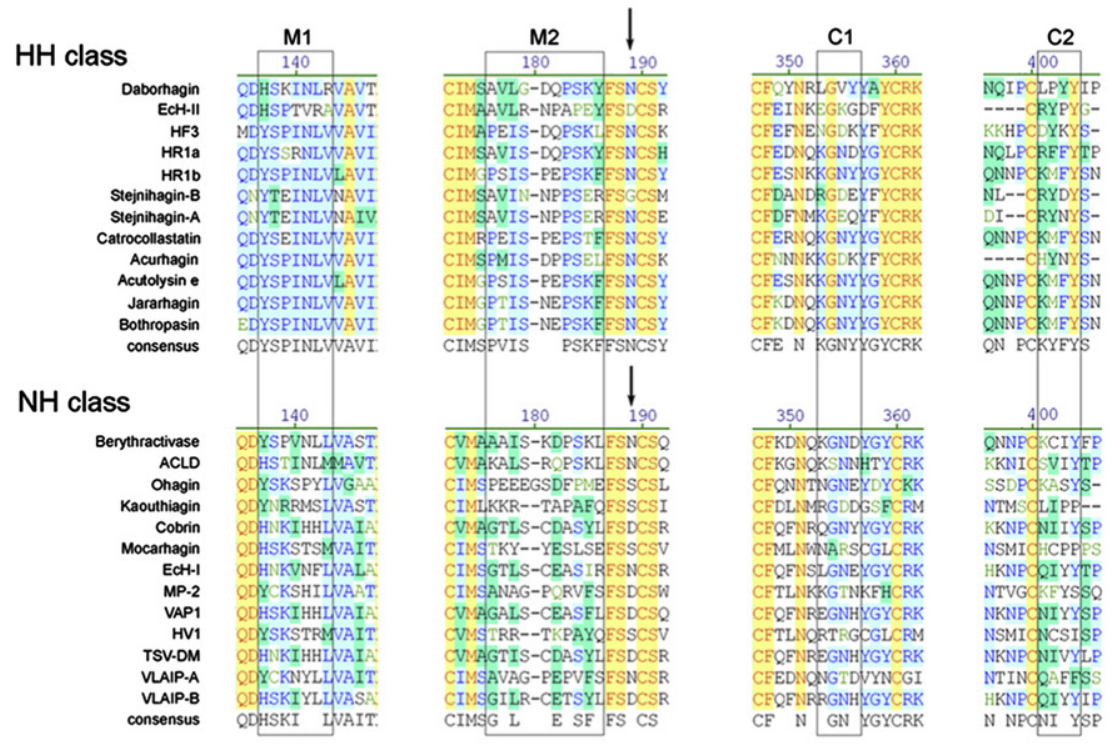

C
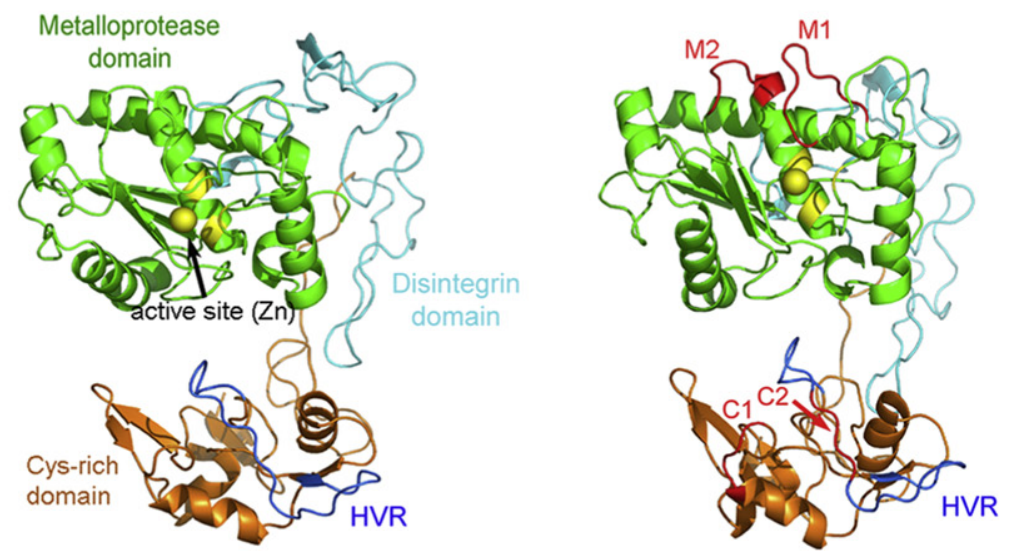

Fig. 6. Analysis of the structural elements responsible for classification of $\mathrm{HH}$ and $\mathrm{NH}$ enzymes. (A) Absolute complexity profiles of $\mathrm{HH}$ and $\mathrm{NH}$ classes are represented as solid and dotted lines, respectively. Four structural motifs (M1, M2, C1, and C2) are indicated by arrows. (B) Sequence alignments of the four motifs (boxed) in both classes. The $N$-glycosylation sites at Asn189 are indicated by arrows. (C) Locations of the four motifs in the 3D model of daborhagin. (Left) The backbones of three structural domains of daborhagin are marked and shown in different colors. HVR loop and zinc-binding residues in the active site are labeled in blue and gold, respectively. Catalytic zinc ion $(\mathrm{Zn})$ is represented as a gold sphere. (Right) Their corresponding locations are highlighted in red in another orientation.

Presumably, the binding of $\mathrm{Mg}^{2+}$ and $\mathrm{Ca}^{2+}$ ions is important for the stability and activity of daborhagin $[29,35]$. However, the only metal ion detected in VRR-73 was $\mathrm{Mg}^{2+}$ in a mol per mol ratio. Additionally, VRR-73 has high arginine esterolytic activity and is strongly inhibited by PMSF [16], which suggests that some esterase might have been included in their purified VRR-73 sample.
Insulin chain B has been a model substrate for studying the cleavage specificities and active site microenvironments of the SVMPs [28]. Daborhagin hydrolyzed insulin chain B at four peptide bonds $\left(\mathrm{His}^{10}-\mathrm{Leu}^{11}, \mathrm{Ala}^{14}-\mathrm{Leu}^{15}, \mathrm{Tyr}^{16}-\mathrm{Leu}^{17}\right.$, and $\mathrm{Phe}^{24}-\mathrm{Phe}^{25}$ ), all of which had a Leu or an aromatic residue at the $\mathrm{P}_{1}{ }^{\prime}$ position. Moreover, the most rapid cleavage bonds contained an Ala or Tyr at the $\mathrm{P}_{1}$ position, different 
from those of weakly hemorrhagic Ht-c and Ht-d (P-I class), which prefer a small residue at the $\mathrm{P}_{1}$ position [36]. In support of this idea is the observation that daborhagin hydrolyzed NFF-2 (cleaving at Ala-Nva) more efficiently than it did FS-1 (cleaving at Gly-Leu) or NFF-3 (cleaving at GluNva). Notably, daborhagin cleaved the bond between His ${ }^{10}$ and $\mathrm{Leu}^{11} \mathrm{Val}^{12}$, but not the bond between $\mathrm{His}^{5}$ and $\mathrm{Leu}^{6} \mathrm{Cys}^{7}(-$ $\mathrm{SO}_{3} \mathrm{H}$ ) in insulin chain-B, which suggested that negatively charged residues at the $\mathrm{P}_{2}^{\prime}$ position were not favored. Taking all these findings together, daborhagin has a substrate specificity toward hydrophobic and less polar amino acid residues at the $\mathrm{P}_{1}, \mathrm{P}_{1}{ }^{\prime}$, and $\mathrm{P}_{2}{ }^{\prime}$ positions.

Molecular phylogeny has been a powerful tool in classifying venom protein families and identifying distinct functional subtypes. Our previous phylogenetic analyses of the P-I (and processed P-II metalloproteinases) clearly revealed three existing subtypes [25]. Recently, two cladograms showed that P-III SVMPs could be divided into P-IIIa, P-IIIb, and P-IIIc subclasses $[2,9]$. With more P-III sequences, our new phylogenetic tree revealed two major subtypes (designated $\mathrm{HH}$ and $\mathrm{NH}$ classes). Consistent with its functional assays, daborhagin belongs to the $\mathrm{HH}$ class and is associated with several of the strongest hemorrhagins. Remarkably, there is no subclass-specific seventh cysteinyl residue in the metalloproteinase domains of daborhagin, EcH-II, HR1a, or HF3 (Figs. 4 and 5). This led us to hypothesize that they constitute another new hemorrhagic group among the $\mathrm{HH}$ members in addition to P-IIIa and P-IIIc. It is noteworthy that these four P-III enzymes and P-IIIc are more hemorrhagic than P-IIIa, and that they contain an average of four $\mathrm{N}$-glycosylation sites while P-IIIa contains only one (Fig. 5). Further investigations of whether these glycosylation sites are important to hemorrhagic activity are needed. By contrast, the NH group is more versatile and further divided into three clusters, possibly with different functions, e.g. inducing endothelial cell apoptosis [7].

Our phylogenetic tree also suggests the co-evolution of paralogous $\mathrm{HH}$ and $\mathrm{NH}$ enzymes in many venom species (Fig. 4), e.g. P. flavoviridis (HR1a, HR1b, HV1) and Crotalus atrox (Catrocollastatin/VAP2B, VAP1). Recent studies $[18,31,37,38]$ have also reported that at least two types of PIIIs with different biological functions are expressed in D. acutus (Acurhagin, AAV1) and V. ammodytes (VaH1, VaH2, Ammodytase) venoms. These paralogous P-IIIs co-expressed in snake venoms might synergistically affect different targets and adapt to the ecology of their prey. Moreover, both P-III paralogs might have evolved before the subfamilies Viperinae and Crotalinae split.

Each member of the P-III SVMPs elicits varying degrees of hemorrhagic potency, and the responsible structural elements are still puzzling $[2,39]$. Since the tree topology clearly classified the P-III SVMPs into two major classes with distinct hemorrhagic potencies, the motifs or residues that distinguish these two classes in the phylogenetic analysis might also be critical in P-III hemorrhagic potencies. Based on this consideration, we identified four conserved motifs in the $\mathrm{HH}$ class, relative to the NH class. Of these, the M1 and M2 motifs are in the metalloproteinase domain and separated by the zinc-binding region and the Met-turn. Both motifs form loop structures around the catalytic site (Fig. 6C). The M1 motif (positions 137-143) serves as a dimer-interface in the P-IIIb subclass [29], and the formation of dimer probably hinders the entrance of substrates to the active site and thus restricts the enzyme specificity. The M2 motif in the HH class contains a conserved proline bracket [40], ${ }^{176} \mathrm{PVISxxP}^{183}$, which has been suggested to form one wall of the extended substrate-binding site of SVMPs [41]. The other two motifs, $\mathrm{C} 1$ and $\mathrm{C} 2$, are in the Cys-rich domain. 3D homologous modeling showed that the $\mathrm{C} 1$ motif forms the hydrophobic ridge and that the $\mathrm{C} 2$ motif is the central part of the hyper-variable region (HVR). Both the $\mathrm{C} 1$ and $\mathrm{C} 2$ motifs have been suggested to constitute a potential protein-protein interaction interface [29]. By contrast, the disintegrin domains of both the $\mathrm{HH}$ and $\mathrm{NH}$ classes are similar in their absolute complexity profiles, which agree with findings that the disintegrin domain might be merely a linker for the other two domains [29]. Collectively, these four structural motifs are probably important determinants for substrate interaction and the P-III hemorrhagic potencies.

The $\mathrm{p} I$ values of HH-class members (average 5.5) were lower than those of NH-class members (average 7.4). By comparing the surface electro-potential of 3D models between more than 10 representative P-III SVMPs (data not shown), we found that most $\mathrm{HH}$ enzymes bore more surface negative charges than NH enzymes did. The importance of this difference remains to be determined. Remarkably, the $N$-glycosylation site at Asn189 (Fig. 6B), on the C-terminal side of the M2 motif, is important for the hemorrhagic potencies of jararhagin [42], and this site is strictly conserved in $\mathrm{HH}-$ but not in $\mathrm{NH}-$ class members.

Fluorogenic peptide substrates have been successfully used for comparing the specificities of SVMPs [36,43]. The synthetic tetrapeptide Abz-Ala-Gly-Leu-Ala-Nba (cleaving at Gly-Leu) has been examined as a good substrate for weakly hemorrhagic Ht-c and Ht-d toxins [36]. Additionally, a 38-kDa non-hemorrhagic metalloproteinase from Rhabdophis tigrinus tigrinus venom has high proteolytic specificities towards FS-1 (cleaving at Gly-Leu) [43]. Here, we found that the highly hemorrhagic P-III enzymes were more active toward three fluorogenic substrates, especially NFF-2 and NFF-3 (Table 1). Further kinetic analyses suggested that the differences between the $\mathrm{HH}$ and NH enzymes for NFF-2 are in both $k_{\text {cat }}$ and $K_{\mathrm{m}}$. Three regions-residues $140-143,176-181$ and 182-185-were found to be related to the active site domains of SVMPs [41,44,45] and located in M1 and M1 motifs. Remarkably, these regions within the $\mathrm{HH}$ members are more hydrophobic (including Ile140, Asn141, Leu142, Val143, Val177, and Ile178) and rigid (Pro139, 176, and 183) (Fig. 6B). Presumably, by favorable interactions with hydrophobic and bulky residues in the substrates (e.g. the $\mathrm{P}_{1}$ and $\mathrm{P}_{1}{ }^{\prime}$ subsites), $\mathrm{HH}$ members might trigger a better induced-fit effect and subsequent transition state stabilization to increase their $k_{\mathrm{cat}}$. Notably, the specificity of the HH enzymes for these fluorogenic substrates appears to be similar to that of the vertebrate hemorrhagic MMP3 (Stromelysin 1) [22,23], which 
may activate several MMP zymogens [46]. Whether daborhagin or other HH-class members activate endogenous MMPs or inflammatory cytokines, which may contribute to local tissue damage, requires further investigation $[47,48]$.

In summary, we purified and characterized daborhagin, a highly hemorrhagic P-III metalloproteinase of Russell's viper's venom, and solved its sequence. Daborhagin has the high proteolytic activity toward fibrinogen, collagen, and fibronectin; this activity appears to be related to the severe bleeding symptoms of its envenomed victims. Moreover, a phylogenetic analysis unraveled the co-evolution of two paralogous P-III classes with different hemorrhagic potencies in the venoms of Viperidae, and daborhagin apparently belongs to the new, highly hemorrhagic subclass. We identified four regions related to the classifications of the P-III SVMPs; detailed studies of their importance to hemorrhagic activities and functional diversities can now begin. Our findings not only unravel the important toxicology of hemorrhagin in specific populations of Daboia, but also provide a good basis for designing rational mutagenesis experiments to study the structure-function relationships of the P-III SVMPs.

\section{Acknowledgments}

We thank Professors Antony Gomes (University of Calcutta, India), Yu-Yen Shu (Kuangxi Medical University, China), and R. David G. Theakston (Liverpool School of Tropical Medicine, UK) for their gifts of venom samples, and Mr. S. Lin for proofreading the manuscript. This work was supported by grants from the National Science Council and Academia Sinica of Taiwan.

\section{References}

[1] H. Takeya, S. Iwanaga, Proteases that induce hemorrhage, in: G.S. Bailey (Ed.), Enzymes from Snake Venom, Alaken, Colorado, 1998, pp. 11-38.

[2] J.W. Fox, S.M. Serrano, Structural considerations of the snake venom metalloproteinases, key members of the M12 reprolysin family of metalloproteinases, Toxicon 45 (2005) 969-985.

[3] J.M. Gutierrez, A. Rucavado, T. Escalante, C. Diaz, Hemorrhage induced by snake venom metalloproteinases: biochemical and biophysical mechanisms involved in microvessel damage, Toxicon 45 (2005) 997-1011.

[4] T. Escalante, J. Shannon, A.M. Moura-da-Silva, J.M. Gutierrez, J.W. Fox, Novel insights into capillary vessel basement membrane damage by snake venom hemorrhagic metalloproteinases: a biochemical and immunohistochemical study, Arch. Biochem. Biophys. 455 (2006) 144-153.

[5] S. Nishida, T. Fujita, N. Kohno, H. Atoda, T. Morita, H. Takeya, I. Kido, M.J. Paine, S. Kawabata, S. Iwanaga, cDNA cloning and deduced amino acid sequence of prothrombin activator (ecarin) from Kenyan Echis carinatus venom, Biochemistry 34 (1995) 1771-1778.

[6] M.B. Silva, M. Schattner, C.R. Ramos, I.L. Junqueira-de-Azevedo, M.C. Guarnieri, M.A. Lazzari, C.A. Sampaio, R.G. Pozner, J.S. Ventura, P.L. Ho, A.M. Chudzinski-Tavassi, A prothrombin activator from Bothrops erythromelas (jararaca-da-seca) snake venom: characterization and molecular cloning, Biochem. J. 369 (2003) 129-139.

[7] I. Tanjoni, R. Weinlich, M.S. Della-Casa, P.B. Clissa, R.F. SaldanhaGama, M.S. de Freitas, C. Barja-Fidalgo, G.P. Amarante-Mendes, A.M. Moura-da-Silva, Jararhagin, a snake venom metalloproteinase, induces a specialized form of apoptosis (anoikis) selective to endothelial cells, Apoptosis 10 (2005) 851-861.
[8] L.C. Wijeyewickrema, M.C. Berndt, R.K. Andrews, Snake venom probes of platelet adhesion receptors and their ligands, Toxicon 45 (2005) 1051-1061.

[9] S.G. Wan, Y. Jin, W.H. Lee, Y. Zhang, Cloning of two novel P-III class metalloproteinases from Trimeresurus stejnegeri venom gland, Toxicon 47 (2006) 465-472.

[10] D.A. Warrell, Snake venoms in science and clinical medicine. 1. Russell's viper: biology, venom and treatment of bites, Trans. R. Soc. Trop. Med. Hyg. 83 (1989) 732-740.

[11] W. Wuster, S. Otsuka, A. Malhotra, R.S. Thorpe, Population systematics of Russell's viper: a multivariate study, Biol. J. Linn. Soc. 47 (1992) 97-113.

[12] R.E. Phillips, R.D.G. Theakston, D.A. Warrell, Y. Galigedara, D.T. Abeysekera, P. Dissanayaka, R.A. Hutton, D.J. Aloysius, Paralysis, rhabdomyolysis and haemolysis caused by bites of Russell's viper ( $\mathrm{Vi}$ pera russelli pulchella) in Sri Lanka: failure of Indian (Haffkine) antivenom, Q. J. Med. 68 (1988) 691-715.

[13] D.A. Warrell, Geographic and intraspecies variation in the clinical manifestations of envenoming by snakes, in: R.S. Thorpe, W. Wuster, A. Malhotra (Eds.), Venomous Snakes, Clarendon Press, Oxford, UK, 1997, pp. 189-204.

[14] Than-Than, R.A. Hutton, Myint-Lwin, Khin-Ei-Han, Soe-Soe, Tin-NuSwe, R.E. Phillips, D.A. Warrell, Haemostatic disturbances in patients bitten by Russell's viper (Vipera russelli siamensis) in Burma, Br. J. Haematol. 69 (1988) 513-520.

[15] N.B. Prasad, B. Uma, S.K. Bhatt, V.T. Gowda, Comparative characterisation of Russell's viper (Daboia/Vipera russelii) venoms from different regions of the Indian peninsula, Biochim. Biophys. Acta 1428 (1999) 121-136.

[16] D. Chakrabarty, K. Datta, A. Gomes, D. Bhattacharyya, Haemorrhagic protein of Russell's viper venom with fibrinolytic and esterase activities, Toxicon 38 (2000) 1475-1490.

[17] I.H. Tsai, H.Y. Tsai, Y.M. Wang, Tun-Pe, D.A. Warrell, Venom phospholipases of Russell's vipers from Myanmar and eastern India-Cloning, characterization and phylogeographic analysis, Biochim. Biophys. Acta 1774 (2007) 1020-1028.

[18] W.J. Wang, T.F. Huang, Purification and characterization of a novel metalloproteinase, acurhagin, from Agkistrodon acutus venom, Thromb. Haemost. 87 (2002) 641-650.

[19] Y. Yamakawa, T. Omori-Satoh, D. Mebs, Hemorrhagic principles in the venom of Bitis arietans, a viperous snake. II. Enzymatic properties with special reference to substrate specificity, Biochim. Biophys. Acta 1247 (1995) 17-23.

[20] S.G. Wan, Y. Jin, W.H. Lee, Y. Zhang, A snake venom metalloproteinase that inhibited cell proliferation and induced morphological changes of ECV304 cells, Toxicon 47 (2006) 480-489.

[21] H. Kondo, S. Kondo, H. Ikezawa, R. Murata, Studies on the quantitative method for determination of hemorrhagic activity of Habu snake venom, Jpn. J. Med. Sci. Biol. 13 (1960) 43-52.

[22] C.G. Knight, F. Willenbrock, G. Murphy, A novel coumarin-labelled peptide for sensitive continuous assays of the matrix metalloproteinases, FEBS Lett. 296 (1992) 263-266.

[23] H. Nagase, C.G. Fields, G.B. Fields, Design and characterization of a fluorogenic substrate selectively hydrolyzed by stromelysin 1 (matrix metalloproteinase-3), J. Biol. Chem. 269 (1994) 20952-20957.

[24] E. Harlow, D. Lane, Immunoblotting, in: E. Harlow, D. Lane (Eds.), Antibodies: A Laboratory Manual, Cold Spring Harbor Laboratory, Cold Spring Harbor, NY, 1988, pp. 471-510.

[25] I.H. Tsai, Y.M. Wang, T.Y. Chiang, Y.L. Chen, R.J. Huang, Purification, cloning and sequence analyses for pro-metalloprotease-disintegrin variants from Deinagkistrodon acutus venom and subclassification of the small venom metalloproteases, Eur. J. Biochem. 267 (2000) 1359-1367.

[26] J. Felsenstein, Confidence limits on phylogenies: an approach using the bootstrap, Evolution 39 (1985) 783-791.

[27] T. Igarashi, S. Araki, H. Mori, S. Takeda, Crystal structures of catrocollastatin/VAP2B reveal a dynamic, modular architecture of ADAM/adamalysin/reprolysin family proteins, FEBS Lett. 581 (2007) $2416-2422$. 
[28] J.B. Bjarnason, J.W. Fox, Snake venom metalloendopeptidases: reprolysins, Methods Enzymol. 248 (1995) 345-368.

[29] S. Takeda, T. Igarashi, H. Mori, S. Araki, Crystal structures of VAP1 reveal ADAMs' MDC domain architecture and its unique C-shaped scaffold, EMBO J. 25 (2006) 2388-2396.

[30] J.B. Bjarnason, J.W. Fox, Hemorrhagic metalloproteinases from snake venoms, Pharmacol. Ther. 62 (1994) 325-372.

[31] A. Leonardi, F. Gubenek, I. Krizaj, Purification and characterisation of two hemorrhagic metalloproteinases from the venom of the long-nosed viper, Vipera ammodytes ammodytes, Toxicon 40 (2002) 55-62.

[32] E.N. Baramova, J.D. Shannon, J.B. Bjarnason, J.W. Fox, Degradation of extracellular matrix proteins by hemorrhagic metalloproteinases, Arch. Biochem. Biophys. 275 (1989) 63-71.

[33] Win-Aung, N. Pakmanee, O. Khow, C. Nambut, V. Sitprija, Cross neutralization of the lethal activities of Myanmar and Thai Russell's viper venoms by Thai and Myanmar antivenoms, J. Nat. Toxins 10 (2001) 335-342.

[34] B.J. Woodhams, S.E. Wilson, B.C. Xin, R.A. Hutton, Differences between the venoms of two sub-species of Russell's viper: Vipera russelli pulchella and Vipera russelli siamensis, Toxicon 28 (1990) 427-433.

[35] F.X. Gomis-Ruth, L.F. Kress, W. Bode, First structure of a snake venom metalloproteinase: a prototype for matrix metalloproteinases/collagenases, EMBO J. 12 (1993) 4151-4157.

[36] J.W. Fox, R. Campbell, L. Beggerly, J.B. Bjarnason, Substrate specificities and inhibition of two hemorrhagic zinc proteases $\mathrm{Ht}-\mathrm{c}$ and $\mathrm{Ht}-$ d from Crotalus atrox venom, Eur. J. Biochem. 156 (1986) 65-72.

[37] W.J. Wang, Purification and functional characterization of AAV1, a novel P-III metalloproteinase, from Formosan Agkistrodon acutus venom, Biochimie 89 (2007) 105-115.

[38] A. Leonardi, J.W. Fox, A. Trampus-Bakijac, I. Krizaj, Ammodytase, a metalloprotease from Vipera ammodytes ammodytes venom, possesses strong fibrinolytic activity, Toxicon 49 (2007) 833-842.

[39] O.H. Ramos, H.S. Selistre-de-Araujo, Comparative analysis of the catalytic domain of hemorrhagic and non-hemorrhagic snake venom metallopeptidases using bioinformatic tools, Toxicon 44 (2004) 529-538.

[40] R.M. Kini, H.J. Evans, A novel approach to the design of potent bioactive peptides by incorporation of proline brackets: antiplatelet effects of ArgGly-Asp peptides, FEBS Lett. 375 (1995) 15-17.

[41] D. Zhang, I. Botos, F.X. Gomis-Ruth, R. Doll, C. Blood, F.G. Njoroge, J.W. Fox, W. Bode, E.F. Meyer, Structural interaction of natural and synthetic inhibitors with the venom metalloproteinase, atrolysin C (form d), Proc. Natl. Acad. Sci. USA 91 (1994) 8447-8851.

[42] L.T. Garcia, L.T. Parreiras e Silva, O.H.P. Ramos, A.K. Carmona, P.A. Bersanetti, H.S. Selistre-de-Araujo, The effect of post-translational modifications on the hemorrhagic activity of snake venom metalloproteinase, Comp. Biochem. Physiol. Part C 138 (2004) 23-32.

[43] K. Komori, M. Konishi, Y. Maruta, M. Toriba, A. Sakai, A. Matsuda, T. Hori, M. Nakatani, N. Minamino, T. Akizawa, Characterization of a novel metalloproteinase in Duvernoy's gland of Rhabdophis tigrinus tigrinus, J. Toxicol. Sci. 31 (2006) 157-168.

[44] K.F. Huang, S.H. Chiou, T.P. Ko, A.H.J. Wang, Determinants of the inhibition of a Taiwan habu venom metalloproteinase by its endogenous inhibitors revealed by X-ray crystallography and synthetic inhibitor analogues, Eur. J. Biochem. 269 (2002) 3047-3056.

[45] W. Gong, X. Zhu, S. Liu, M. Teng, L. Niu, Crystal structures of acutolysin A, a three-disulfide hemorrhagic zinc metalloproteinase from the snake venom of Agkistrodon acutus, J. Mol. Biol. 283 (1998) 657-668.

[46] R. Visse, H. Nagase, Matrix metalloproteinases and tissue inhibitors of metalloproteinases: structure, function, and biochemistry, Circ. Res. 92 (2003) 827-839.

[47] A.M. Moura-da-Silva, G.D. Laing, M.J. Paine, J.M. Dennison, V. Politi, J.M. Crampton, R.D.G. Theakston, Processing of pro-tumor necrosis factor-alpha by venom metalloproteinases: a hypothesis explaining local tissue damage following snake bite, Eur. J. Immunol. 26 (1996) 2000-2005.

[48] A. Rucavado, J. Núñez, J.M. Gutiérrez, Blister formation and skin damage induced by BaP1, a haemorrhagic metalloproteinase from the venom of the snake Bothrops asper, Int. J. Exp. Pathol. 79 (1998) 245-254.

[49] M.T. Assakura, A.P. Reichl, F.R. Mandelbaum, Comparison of immunological, biochemical and biophysical properties of three hemorrhagic factors isolated from the venom of Bothrops jararaca (JARARACA), Toxicon 24 (1986) 943-946.

[50] M.J. Paine, H.P. Desmond, R.D. Theakston, J.M. Crampton, Purification, cloning, and molecular characterization of a high molecular weight hemorrhagic metalloprotease, jararhagin, from Bothrops jararaca venom, J. Biol. Chem. 267 (1992) 22869-22876.

[51] J. Hamako, T. Matsui, S. Nishida, S. Nomura, Y. Fujimura, M. Ito, Y. Ozeki, K. Titani, Purification and characterization of kaouthiagin, a von Willebrand factor-binding and -cleaving metalloproteinase from Naha kaouthia cobra venom, Thromb. Haemost. 80 (1998) 499-505.

[52] H. Takeya, K. Oda, T. Miyata, T. Omori-Satoh, S. Iwanaga, The complete amino acid sequence of the high molecular mass hemorrhagic protein HR1B isolated from the venom of Trimeresurus flavoviridis, J. Biol. Chem. 265 (1990) 16068-16073.

[53] K. Trummal, K. Tõnismägi, E. Siigur, A. Aaspõllu, A. Lopp, T. Sillat, R. Saat, L. Kasak, I. Tammiste, P. Kogerman, N. Kalkkinen, J. Siigur, A novel metalloprotease from Vipera lebetina venom induces human endothelial cell apoptosis, Toxicon 46 (2005) 46-61. 